\title{
Research on Process Innovation of Oxidation Coating on Biomedical Titanium Alloy based on Inventive Principles of TRIZ
}

\author{
CH.Q. GAO \& J.H. SUN
}

School of Mechanical Engineering, University of Jinan, No.336 Nanxinzhuang West Road, Jinan, Shandong, P.R.China

Y. WAN

School of Mechanical Engineering, Shandong University, No.73 Jingshi Road, Jinan, Shandong, P.R.China

ABSTRACT: Cutting is one of the most widely used machining technologies in the mechanical industry. Metal cutting is a complex technology process and innovation methodology is urgently for its innovation TRIZ is a technology innovation method of Soviet Union. Conflict tools of TRIZ are applied to the analysis process of machining technology of titanium alloy with standardize the description of conflict by 39 engineering parameters. With the computer-aided innovation system and corresponding inventive principles, the project of the process innovation of oxidation coating on biomedical titanium alloy is finished. The significance of TRIZ in promoting technology innovation is illustrated.

KEYWORD: TRIZ, technology innovation; biomedical titanium alloy; oxidation coating

\section{APPLICATION OF BIO-MEDICAL TITANIUM ALLOY}

The oxidation coating of biomedical titanium alloy material is critical to its corrosion resistance and biocompatibility. When the biomedical titanium alloy, such as a new-type titanium alloy TLM (Ti$5 \mathrm{Zr}-3 \mathrm{Sn}-5 \mathrm{Mo}-15 \mathrm{Nb}$ ), is implanted into body, it would be affected by various body fluids, soft tissues, bones, etc. The current biomedical titanium alloy should be treated in surface before implantation because material corrosion led to negative impacts in fluid environment, such as allergic reactions, inflammatory response, etc. The generated oxidation coating has a lot of benefits, such as the advantages of low-activity with biological molecules, low toxicity, low solubility in water, obvious anti-inflammatory effects and shielding metal ion dissolution, which made titanium alloy widely used in biomedical materials.

In fluid environment, corrosion of materials in body fluids easily led to allergic reactions and inflammatory response. The excellent corrosion resistance and biocompatibility of titanium alloy mainly due to the oxidation coating of its surface.

The dissolved substances include electrolytes (such as $\mathrm{KCl}$ and $\mathrm{NaCl}$ ) which dissociated into positive and negative ions in body fluids. There is also non-electrolytes (such as glucose, urea, creatinine, etc). There are various body fluids (such as blood, saliva, lymph, joint lubricants, etc) in different parts of the body. In fluid environment, corrosion of materials in body fluids easily led to allergic reactions and inflammatory response. The excellent corrosion resistance and biocompatibility of titanium alloy mainly due to the oxidation coating of its surface.

The surface treatment of oxidation coat forming includes mainly anodic oxidation, micro-arc oxidation, atmosphere heating oxidation and chemical processing method. After dealing with surface, the thickness of oxidation film was 10 20um, as shown in Figure 1 [1].

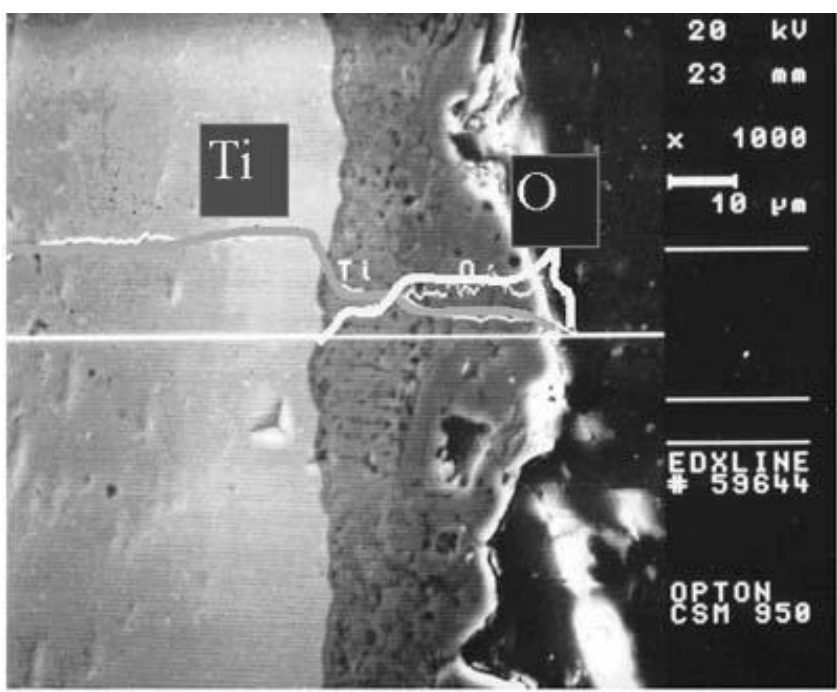

Figure 1. Micro-arc oxidation coating of titanium alloy

Machining is still one of the most widely used manufacturing processes at present and in the future. With the development of auto industry, aerospace, 
energy, molds, electron and biological materials, the energy levels of material, which is going to be processed, are improved constantly. High strength, high toughness and difficult machining materials appear constantly. Machining is developing to high speed and efficiency, better surface integrity, intelligence and environmental protection. Cutting procedure not only need to achieve a nice processing accuracy, but also reach a good surface integrity. Therefore, cutting tools and procedures should meet the requirement of machined parts on fighting fatigue, corrosion resistance, wear-ability, etc. The goal is to save energy, reduce consumption and meet the requirements of machined parts on longer life expectancies and total life circle. The developmental tend of cutting procedure should be more to ensure and intensify surface properties than take sharp of parts surface [2-3].

Because of the complexity of the machining process, the instruction of efficient technology innovation theory is necessary for realizing the innovation of cutting procedure.

\section{TRIZ}

TRIZ is theory of inventive problem solving and the core content of innovation curriculum. It is developed upon the basis of research that Altshuller and his colleagues analyzed nearly 2.5 million invention patents in the world. After summing the knowledge principle and law of subject areas that were used in the invention, the new theory system for innovation was created [4].

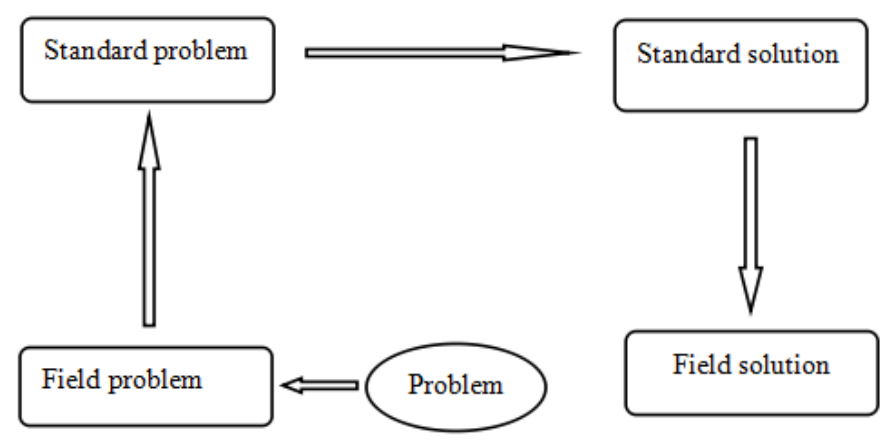

Figure 2. The process of innovation design based on TRIZ

The problem solving tools of TRIZ theory can be divided into two groups. The first one is the analysis tool. This tool help engineers to define and describe the problem and assist the analysis process, which include ARIZ, Field-Substance analysis, etc. The other one is the knowledge base tool. This tool is derived from accumulation and arrangement of human innovation experience and knowledge, which include 40 invention principles, Separation principle, effect, etc. The process of solving problem in application of analysis tool and knowledge base tool is to transform domain problem into standard question, then transform the standard solution into field solution [5].

The process of innovation design based on TRIZ is shown in Figure 2.

\subsection{Conflict}

Conflict is a common inventive problem. TRIZ theory insists that the core of inventive problem is to resolve conflicts. If the design has not solved the conflict it is not the really innovation design. The conflicts of TRIZ theory mainly include technology conflict and physic conflict. Solution to the problem based on TRIZ theory is different from the traditional compromise. When selecting the conflict parameters $\mathrm{A}$ and $\mathrm{B}$. The solution should not only improves the quality of parameter A, but also not affects the quality of parameter B. It is actually a process of solving conflict.

\subsection{Solution and inventive principle of conflict}

TRIZ theory utilizes 40 inventive principles to solve contradiction and standardizes the description of conflict with 39 general engineering parameters. Conflict matrix is a $40 \times 40$ matrix. And its first line and first column are standard engineering parameters of the serial numbers. Get rid of the first line and first column, the rest of the 39 lines and 39 columns constituted a matrix. Its elements is a set of numbers or empty, and this group of numbers represent inventive principle sequence numbers of resolving corresponding conflict.

When matrix with conflict is used, the fist step is to identity technology conflict aimed at specific problem and describe the technology conflict with two standard engineering parameters. Then the available inventive principle by standard engineering parameters in the conflict matrix can be determined.

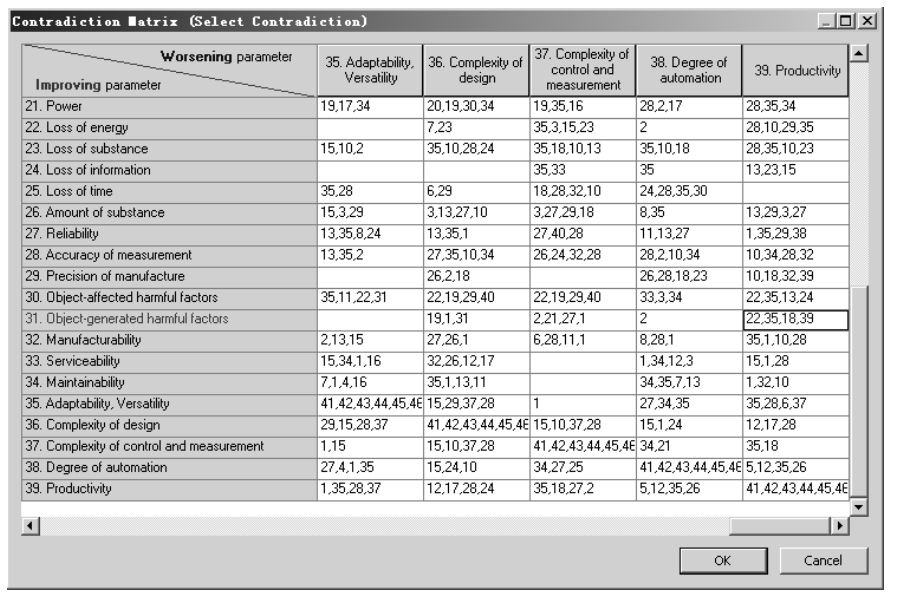

Figure 3. Conflict matrix

Conflict matrix is shown in Figure 3. The process of conflict solving is shown in Figure 4. 


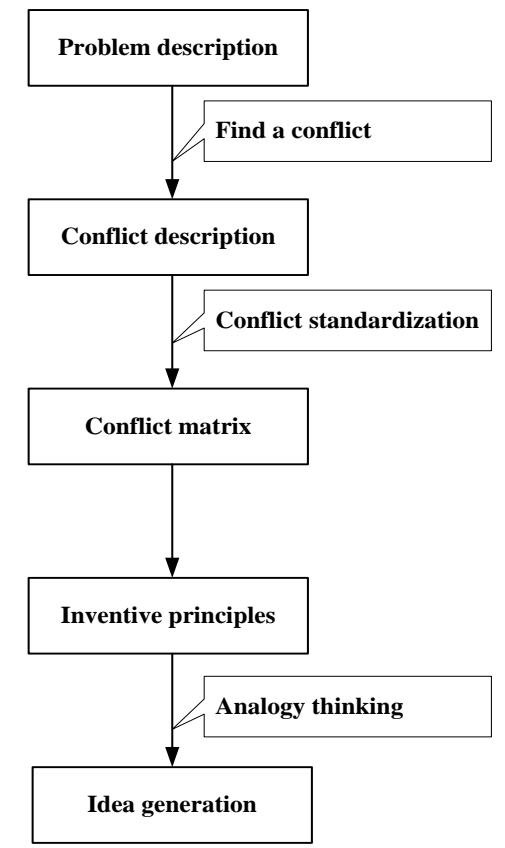

Figure 4. Process of conflict solving

\section{RESEARCH ON INNOVATION DESIGN OF OXIDATION COATING ON BIOMEDICAL TITANIUM ALLOY BASED ON TRIZ}

\subsection{The analysis of existing technology solutions}

In the computer-aided innovation system Pro/Innovator, the scheme of oxidation coating innovation craft on biomedical titanium alloy was gotten.

At present, the oxidation coating on biomedical titanium alloy is formed after finishing machining process. This process is inefficient and higher-cost. Besides, the technology chain is long, and the equipment is complex. The advantage of existed technical process is the mature technology of oxidation film formation and stable quality. At the same time, the long technology chain and the complexity of process equipment led to a lower production efficiency. This is a typical technology conflict. The solution can be found with inventive principle.

Table 1. The suggestion of the principle 22.

\begin{tabular}{|c|c|}
\hline Principles & Suggestion \\
\hline \multirow{3}{*}{$\begin{array}{l}\text { Conversion of Harm into } \\
\text { Benefit }\end{array}$} & $\begin{array}{l}\text { 1. Use harmful factors (particular, effects harmful to the environment or surrounding) a different way } \\
\text { to achieve a positive effect. }\end{array}$ \\
\hline & 2. Eliminate the primary harmful action by adding it to another harmful action to resolve the problem. \\
\hline & 3. Amplify a harmful factor to such a degree that it is no longer harmful \\
\hline \multirow{4}{*}{ Reverse } & 1. Change an object`s physical state (e.g. to a gas, liquid, or solid). \\
\hline & 2. Change the concentration or consistency. \\
\hline & 3. Change the degree of flexibility. \\
\hline & 4. Change the temperature. \\
\hline \multirow{4}{*}{ Changes in Parameters } & 1. change an object`s physical state (e.g. to a gas, liquid, or solid) \\
\hline & 2. Change the concentration or consistency \\
\hline & 3. Change the degree of flexibility \\
\hline & 4. Change the temperature \\
\hline \multirow{2}{*}{ Intermediary Substance } & 1. Use an intermediate carrier article or intermediary process \\
\hline & 2. Merge one object temporarily with another (which can be easily removed) \\
\hline
\end{tabular}

First, the 30th engineering parameter (Objectaffected harmful factors) is selected as tuning better parameter of the existing technology system. Then the 39th engineering parameter (productivity) is chosen as the deterioration parameter of the existing technology system. As shown in Figure 5.

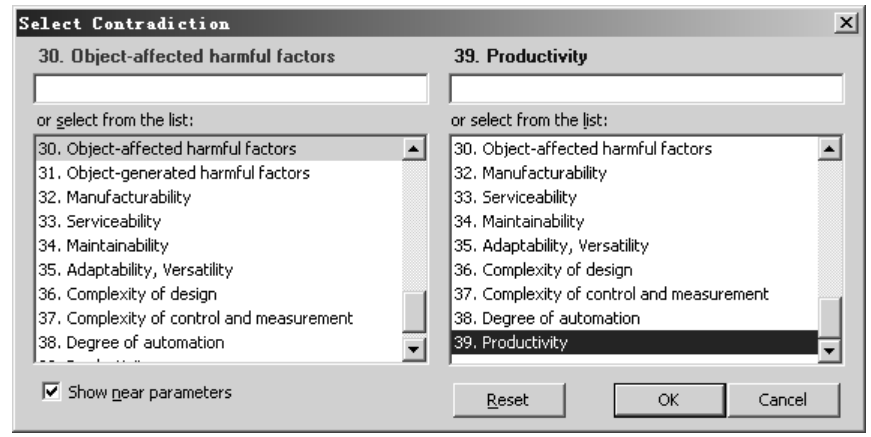

Figure 5. Selected engineering parameters

By the conflict matrix, the certain inventive principles can be we can determined and be used to solve the conflict. As shown in Figure 6.

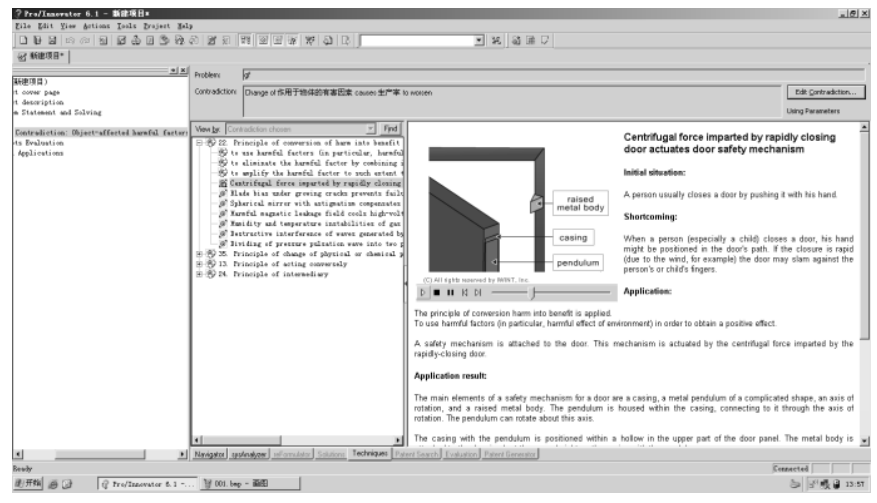

Figure 6. The computer-aided innovation system

The Pro/Innovator system recommended several inventive principles, such as "conversion of harm into benefit", "changes in parameters", "reverse", "intermediary substance", etc. The suggestions of "conversion of harm into benefit" is shown in Table1. 
Among those four inventive principles, "conversion of harm into benefit" is the most instructive one for this problem situation. This inventive principle and the corresponding cases are the important information to study.

The case shows centrifugal force imparted by rapidly closing door actuates door safety mechanism. When a person (especially a child) closes a door, his hand might be positioned in the door's path. If the closure is rapid (duo to the wind, for example) the door may slam against the person's or child's fingers.

The principle of conversion harm into benefit is applied. To use harmful factors (in particular, harmful effect of environment) in order to obtain a positive effect.

A safety mechanism is attached to the door. This mechanism is actuated by the centrifugal force imparted by the rapidly-closing door.

The main elements of a safety mechanism for a door are a casing, a metal pendulum of a complicated shape, an axis of rotation, and a raised metal body. The pendulum is housed within the casing, connecting to it through the axis of rotation. The pendulum can rotate about this axis.

This case shows an inspiration of use the harmful element in the environment into benefit. This may be the key idea for the problem solving.

\subsection{The innovation design process}

At present, the cutting heat of cutting process is one of the main negative factors which affect the tool life. In order to reduce the cutting temperature of titanium alloy cutting process and improve the tool life, liquid coolant is usually used in the process of mental cutting. But the chloride ion in liquid coolant is harmful to human health. The cutting heat may be the harmful element to be considered to be converted into benefit.

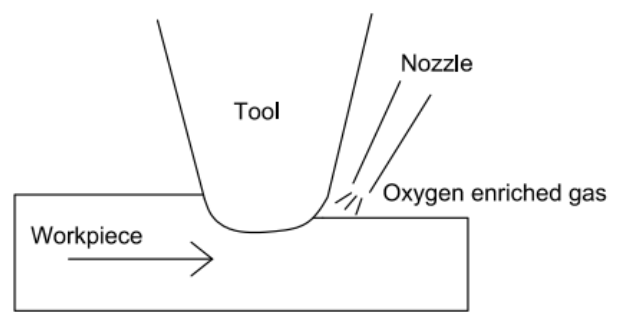

Figure 7. The metal cutting process

According to the solution of "conversion of harm into benefit", a new method of machining process is put forward. In the dry machining process of biomedical titanium alloy, it is an initiative idea to control and use of heat heating on the surface formation of oxidation film. It can substitute or partly replace the subsequent surface oxidation treatment and greatly improve the efficiency and reduce costs. As shown in Figure 7.

\section{CONCLUSTION}

(1) Machining is developing in the direction of high-speed and efficient, Intelligent, environmental protection. Widely used technology innovation method and various fields' knowledge had significant promotion effect on promoting innovation of metal cutting process. TRIZ provided a technological innovation system for solving conflict problem, predicting problem, analog problems, etc. TRIZ theory could make contribution to the realization of technology innovation.

(2) In the dry machining process of biomedical titanium alloy, the initiative to control and use of heat heating on the surface formation of oxidation film is gotten. When it can substitute or partly replace the subsequent surface oxidation treatment, greatly improving the efficiency and reducing costs can be realized. This design scheme had important significance on enriching machining theory and shortening the process chain and developing new cutting tools. Many details are still need further researched in the near future.

\section{ACKNOWLEDGEMENTS}

This work was financially supported by Science and Technology Development Plan Project of Shandong Province (2014GGX106003), Large Equipment Upgrade Project of Shandong Province (2012SJGZ15).

\section{REFERENCES}

[1] http://lenp.bnu.edu.cn/lenp/kxyj/whyh/DengLiZiTiWeihu.h tm

[2] AI Xing, LIU Zhanqiang. High speed cutting technology, National Defend Industry Press, Beijing, 2003.

[3] Wei Yanping. Study on the cyto-biocompatibility of two neotype implant titanium alloys and the alloy with surface treatments, Dissertation for Master Degree, Xian, Fourth Military Medical University, 2007.

[4] VICTOR F, EUGENE R, Innovation on Demand, Cambridge University Press, Cambridge, 2007.

[5] Semyon D. Savransky, Engineering of creativity, CRC Press, Florida, 2000. 